\title{
Volkswirtschaftliche und raumordnungspolitische Aspekte der Widmungsabgabe nach dem Entwurf zur Novelle zum Tiroler Raumordnungsgesetz
}

\author{
Robert Wieser • Wilfried Schönbäck
}

Eingegangen: 23. Dezember 2010 / Angenommen: 14. Juli 2011 / Online publiziert: 11. August 2011

(C) Springer-Verlag 2011

\begin{abstract}
Zusammenfassung Ein im Sommer 2010 von der Tiroler Landesregierung präsentierter Entwurf zur Novelle des Tiroler Raumordnungsgesetzes sah unter anderem die Einführung einer Widmungsabgabe in Höhe von $10 \%$ des Widmungsgewinnes bei aufwertenden Widmungen vor. Das Aufkommen aus der Widmungsabgabe sollte dem Tiroler Bodenfonds zufließen, welcher dazu angehalten gewesen wäre, die ihm zufließenden Gelder zum Ankauf zusätzlicher Grundstücke zur Unterstützung des sozialen Wohnbaus in Tirol einzusetzen. Der Entwurf ist in Folge des starken Widerstandes vor allem der Gemeinden nicht beschlossen worden. Der vorliegende Beitrag beschäftigt sich mit den volkswirtschaftlichen und raumordnungspolitischen Aspekten der vorgeschlagenen Regelungen und Maßnahmen. Untersucht werden bodenökonomische, wohnungsökonomische, institutionelle und verteilungspolitische Fragen.
\end{abstract}

Schlagwörter Raumplanung · Flächenwidmung · Widmungsabgabe $\cdot$ Bodenmarkt $\cdot$ Allokation $\cdot$ Verteilung

Dr. R. Wieser $(\bowtie) \cdot$ Prof. Dr. W. Schönbäck

Department für Raumentwicklung, Infrastruktur- und

Umweltplanung, Fachbereich Finanzwissenschaft

und Infrastrukturpolitik, Technische Universität Wien,

Resselgasse 5/2/2, 1040 Wien, Österreich

E-Mail: robert.wieser@tuwien.ac.at

Prof. Dr. W. Schönbäck

E-Mail: wilfried.schoenbaeck@tuwien.ac.at
Economic and Regional Planning Policy Aspects of the Zoning Levy Under the Draft Amendment to the Tyrolean Regional Planning Act

\begin{abstract}
A draft amendment of the Tyrolean Regional Planning Act set out in summer 2010 by the Tyrolean Government, inter alia, scheduled a levy of $10 \%$ on land value uplifts as a consequence of rezoning. The revenues from the zoning levy should have gone to the Tyrolean Land Fund, which essentially would have been encouraged to use the funds to buy additional land in order to support social house building in Tyrol. Actually, the draft was not adopted because of the strong resistance shown particularly by the municipalities. This paper deals with the economic and regional planning policy aspects of the proposed schemes and measures. It examines land economic, housing economic, institutional and distributional issues.
\end{abstract}

Keywords Regional planning · Zoning · Zoning levy · Land markets $\cdot$ Allocation $\cdot$ Distribution

\section{Einleitung}

Seit Jahren wird in Tirol über eine Novelle zum Raumordnungsgesetz (TROG) diskutiert, die dazu beitragen soll, den hohen Baulandüberhang entweder zu mobilisieren und einer Bebauung zuzuführen oder durch Rückwidmungen abzubauen. Im Jahr 2008 waren in Tirol rund 21.340 ha entweder bebaut oder als Bauland ausgewiesen, und jedes Jahr kommen über 100 ha an neu gewidmeten Flächen hinzu. Ein Großteil der Baulandreserven befindet sich in zentralen Lagen und wäre daher ideal für die weitere Besiedelung. Doch kann ein großer Teil dieser Flächen derzeit nicht aktiviert werden. Rund 2.100 ha Bauland, also 
rund $10 \%$, werden gehortet, und die steigende Nachfrage zwingt zu Neuwidmungen an weniger geeigneten Standorten (Nindler 2010). Der Mobilisierung der gehorteten Flächen gilt daher zunehmend die Aufmerksamkeit der Tiroler Raumordnungspolitik.

Im Juli 2010 hat die Tiroler Landesregierung einen Entwurf zur Novelle des Raumordnungsgesetzes vorgelegt (TROG-Novelle 2010). Zwei Kernpunkte des Entwurfs waren die Möglichkeit von Baulandrückwidmungen und die Erhebung einer „Widmungsabgabe“ in jenen Fällen, in denen sich durch Umwidmung von Grundstücken im Vergleich zur vorherigen Widmung eine Steigerung des ortsüblichen Verkehrswertes ergibt. Die Widmungsabgabe war als ausschließliche Landesabgabe in Höhe von $10 \%$ der Steigerung des ortsüblichen Verkehrswertes konzipiert. Deren Aufkommen sollte nach Abzug der vom Land in Höhe von $6 \%$ des Abgabenaufkommens veranschlagten Verwaltungsaufwendungen dem Tiroler Bodenfonds zufließen. Die Steigerung des ortsüblichen Verkehrswertes sollte auf der Grundlage eines Gutachtens eines Sachverständigen für Liegenschaftsbewertungen ermittelt werden. Zur Entrichtung der Widmungsabgabe sollte der Grundstückseigentümer, im Fall des Bestehens eines Baurechtes der Bauberechtigte, verpflichtet sein. Nicht abgabepflichtig sollte der Tiroler Bodenfonds sein. ${ }^{1}$

Den Verfassern der Novelle war bewusst, dass das Aufkommen aus der Widmungsabgabe jährlich hohen Schwankungen unterliegen würde, weil erstens die Grundstückspreise landesweit stark differieren und zweitens das Widmungsgeschehen in räumlicher Hinsicht nicht vorhersagbar ist und unterschiedlich verläuft. Im langjährigen Durchschnitt jedoch ist in Tirol von etwa 1.200 Widmungsfällen pro Jahr auszugehen. Eine grobe Schätzung für das Jahr 2008 ergab, dass die Widmungsgewinne im Baulandbereich (Wohn- und Mischgebiete, Gewerbe- und Industriegebiete, ohne Sonderflächen) rund 104 Mio. $€$ betragen haben dürften (TROG-Novelle, Erläuternde Bemerkungen 2010: 12). Der Abgabenertrag der Widmungsabgabe aus diesen Baulandsegmenten hätte demnach rund 10,4 Mio.€ betragen, wovon rund $624.000 €$ vom Bodenfonds an das Land geleistet worden wären. ${ }^{2}$

\footnotetext{
${ }^{1} \S 36 \mathrm{a}(7)$ der Novelle enthielt eine Symmetrieregelung, wonach bei Rückwidmungen zur vormals bestehenden Widmung eine erhobene Widmungsabgabe, auf Antrag des Grundstückseigentümers, wieder zurückgezahlt werden sollte. Weiters sollte eine erhobene Widmungsabgabe anteilsmäßig zurückbezahlt werden, wenn sich durch eine neuerliche Widmung (Umwidmung) eine Minderung des ortsüblichen Verkehrswertes ergibt. Für den Rückzahlungsanspruch sollte eine spezielle Verjährungs- und Abschlagsregelung (§ 36a (8)) gelten. Für die Widmungsabgabe samt Nebengebühren sollte auf dem jeweiligen Grundstück ein gesetzliches Pfandrecht haften.

${ }^{2}$ Die Widmungsgewinne im Fall von Umwidmungen in Sonderflächen (beispielsweise Einkaufszentren) waren aufgrund der hohen Wertdifferenzen unterschiedlicher Nutzungen in diesem Bereich pauschal
}

In diesem Beitrag werden die wichtigsten Wirkungen und Probleme einer Widmungsabgabe aus bodenökonomischer und finanzwissenschaftlicher Sicht qualitativ untersucht. Im Einzelnen wird folgenden Fragen nachgegangen:

- Was ist ein Widmungsgewinn? (Kap. 3)

- Wie werden widmungsbedingte Steigerungen von Grundstückswerten ermittelt? (Kap. 4.2)

- Unter welchen Bedingungen wirkt die Widmungsabgabe baulandmobilisierend? (Kap. 4.3)

- Worin bestehen die direkten Verteilungswirkungen der Widmungsabgabe? (Kap. 4.4)

- Inwiefern und in welchem Ausmaß stärkt die Widmungsabgabe den Bodenfonds? - Allokativer Aspekt (Kap. 4.5)

- In welchem Verhältnis steht die Widmungsabgabe zu anderen steuerpolitischen Maßnahmen und Instrumenten? (Kap. 4.6)

- In welchem Verhältnis steht die Widmungsabgabe zu anderen raumplanerischen Maßnahmen und Instrumenten? (Kap. 4.7)

Vor der Abhandlung dieser Fragen wird in Kap. 2 untersucht, was die hohen Baulandpreise in Tirol verursacht und ob Baulandhortung ein flächendeckendes Problem dieses Bundeslandes ist.

\section{Zur Bodenproblematik in Tirol}

\subsection{Was verursacht die hohen Baulandpreise in Tirol?}

Die Baulandpreise in den Tiroler Gemeinden zählen zu den höchsten in Österreich. Nur die österreichischen Kernstädte, die Region Wien und Umgebung, sowie Teile Salzburgs und Vorarlbergs und einige wenige begünstigte Lagen (z. B. Seelagen, Top-Touristenorte) verzeichnen ähnlich hohe oder noch höhere durchschnittliche Baulandpreise. Das Niveau der Baulandpreise ist allerdings nach Bezirken recht unterschiedlich. Es reicht für baureife Grundstücke der Bauklasse I (zum Bau von Eigenheimen und Reihenhäusern) von durchschnittlich $60 €$ pro $\mathrm{m}^{2}$ bis weit über $200 €$ pro $\mathrm{m}^{2}$ (vgl. Abb. $1^{3}$ ).

Die Höhe der Baulandpreise und ihre Entwicklung sind letztlich Ausdruck der effektiven Nachfrage nach Bauland in Relation zum effektiven Baulandangebot. $\mathrm{Zu}$ den wichtigsten Nachfragefaktoren zählen bekanntlich der Stand und die Entwicklung von Bevölkerung und Einkommen. Die Wohnbevölkerung Tirols wächst seit Jahrzehnten überdurch-

nicht schätzbar (TROG Novelle, Erläuternde Bemerkungen 2010: 12). Es dürfte sich aber um eine ähnliche Größenordnung wie im Wohnbausegment handeln.

${ }^{3}$ Aus der Quelle musste die ungenaue Klassifizierung der Grundstückspreise übernommen werden. 
Abb. 1 Durchschnittliche Grundstückspreise pro $\mathrm{m}^{2}$ für baureife Grundstücke der Bauklasse 1 nach politischen Bezirken. (Quellen: Zeitschrift Gewinn 2010, Grundstückspreise in Österreich. Grafik: Institut für Stadt- und Regionalforschung, Technische Universität Wien)

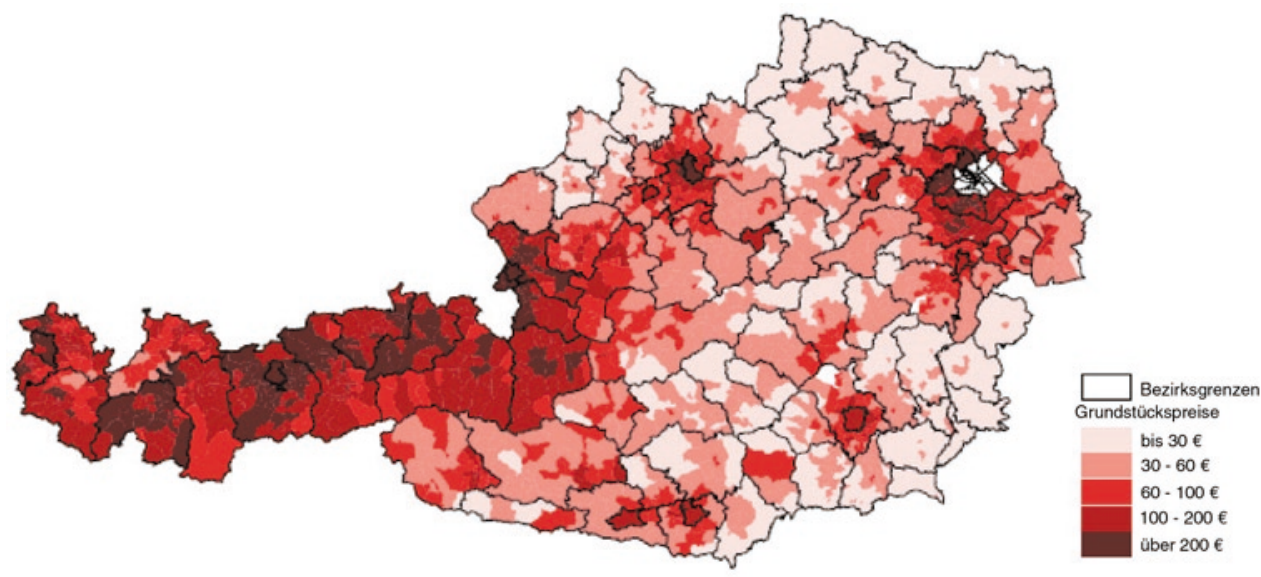

schnittlich stark. Zwischen 1981 und 2009 ist die Bevölkerungszahl um 19,5\% angestiegen. Nur Vorarlberg hatte mit 20,5 \% einen noch größeren relativen Bevölkerungszuwachs (österreichweit: 10,5\%). Seit 2001 hat die Bevölkerung in Tirol um 4,6 \% zugenommen, die dritthöchste Rate nach Wien (8,3\%) und Vorarlberg (4,8 \%) (Statistik Austria 2010e). Für die nächsten beiden Jahrzehnte (bis 2030) geht die Hauptvariante der Bevölkerungsprognose der Statistik Austria von einem weiteren Bevölkerungszuwachs von etwa 45.000 Personen aus. Dies entspräche einem weiteren Zuwachs von 6,5\% binnen 20 Jahren (Statistik Austria 2010f).

Auch im Bereich der Einkommen verzeichnete Tirol überdurchschnittliche Wachstumsraten. Das regionale Bruttoinlandsprodukt pro Kopf hat nominell zwischen 1995 und 2007 um 65,4 \% zugenommen (österreichweit: 55,1 \%). Die verfügbaren Einkommen der privaten Haushalte pro Person sind im gleichen Zeitraum nominell mit 45,8 \% stärker gestiegen als in den meisten anderen Bundesländern. Allerdings lag Tirol beim Niveau der verfügbaren Einkommen pro Person mit $19.100 €$ im Jahr 2007 nur an viertletzter Stelle unter den österreichischen Bundesländern (Statistik Austria 2010a).

Ein dritter und speziell in Tirol sehr wichtiger Nachfragefaktor ist der Tourismus. Tirol ist das Bundesland mit den meisten Beherbergungsbetrieben und -betten in Österreich, sowohl in absoluten als auch in relativen Zahlen. Pro 1.000 Einwohner hatte Tirol im Jahr 2009496 Betten in Tourismusbetrieben aufzuweisen. Nur Salzburg kam mit 388 Betten in die Nähe des Tiroler Werts. Vom Tourismus geht ein starker Nachfragedruck am Bodenmarkt aus. Dies betrifft nicht nur die Beherbergung, sondern die gesamte touristische Infrastruktur. Sowohl die Anzahl der Betriebe als auch die Anzahl der Betten in Tirol sind seit 2001 zwar leicht rückläufig, in zentralen Touristenorten mit tendenziell hohen Bodenpreisen zeigt sich aber eine andere Entwicklung. Beispielsweise ist die Anzahl der Beherbergungs- und Gastronomiebetriebe zwischen 2001 und 2006 in Kitzbühel von 169 auf 192 und in St. Anton von 222 auf 267 angestiegen (Statistik Austria 2010b).

Auf der Angebotsseite des Bodenmarktes gibt es herausragende topographische Beschränkungen. Im Jahr 2008 waren nur 11,9 \% der Fläche Tirols als Dauersiedlungsraum ausgewiesen. Damit liegt Tirol weit hinter dem bezüglich der Beschränktheit des Bodenangebotes nächstgelegenen Bundesland Vorarlberg mit 20,8 \%. Österreichweit liegt der Durchschnitt bei 38 \%. Zum Stichtag 31. Dezember 2008 betrug der Bestand an gewidmetem Bauland 21.343 ha. Dies entsprach einem Anteil von etwa 14,3\% am Dauersiedlungsraum Tirols (Öggl 2010: 1).

Andererseits gibt es einen Stand an Baulandhortungen von rund $10 \%$, die nicht Teil des Baulandangebotes sind. Zum Teil und vor allem bezüglich zentraler Gunstlagen wird daher auch die Baulandhortung für das hohe Bodenpreisniveau verantwortlich gemacht.

\subsection{Sind Baulandhortungen ein flächendeckendes Problem} der Tiroler Bodenmärkte?

Die Frage der wohnungswirtschaftlichen und der darüber hinausgehenden regionalwirtschaftlichen Wirkungen der Baulandhortung ist nicht leicht $\mathrm{zu}$ beantworten. Einige objektive Fakten mögen dazu Hinweise geben. Der Wohnungsbestand (Anzahl der Wohnungen) in Tirol ist zwischen 1971 und 2001 um $90 \%$ angestiegen. Österreichweit lag der Anstieg bei $45 \%$, in Vorarlberg bei $92 \%$. Zwischen 1991 und 2001 hatte Tirol mit $+22 \%$ sogar den stärksten Anstieg (österreichweit: $+14 \%$ ). Nach jüngsten Daten der 
Statistik Austria lag die Wohnbaurate Tirols in den Jahren 2002 und 2005 bis 2009 mit durchschnittlich 7,9 pro Jahr gebauten Wohnungen je 1.000 Einwohnern gemeinsam mit Vorarlberg am höchsten, weit über dem Österreichdurchschnitt von 5,6 Wohnungen je 1.000 Einwohnern (Statistik Austria 2010c). Die Bautätigkeit insgesamt erscheint daher von der Baulandhortung wenig betroffen. Andererseits hängt die Beantwortung der Frage der Wirkungen spekulativer Baulandhortung nicht nur davon ab, wieviel gebaut werden kann, sondern auch davon, wo gebaut werden kann, welche Folgen sich für die Siedlungsentwicklung ergeben und wie die Baulandpreise auf die Wohnungskosten durchschlagen.

Der durchschnittliche Wohnungsaufwand ${ }^{4}$ nach Bundesländern gemäß Statistik Austria ist für Tirol nicht höher als der österreichische Durchschnitt: Der durchschnittliche Wohnungsaufwand pro Eigentumswohnung lag mit $288 €$ im Jahr 2009 in Tirol nur knapp über dem Durchschnittswert für Österreich von $284 €$. Der durchschnittliche Wohnungsaufwand pro $\mathrm{m}^{2}$ in Eigentumswohnungen lag mit 3,37€ sogar unter dem Durchschnitt von $3,43 €$. Der durchschnittliche Wohnungsaufwand pro Mietwohnung (entgeltlich bewohnte Hauptwohnsitzwohnungen) lag mit $406 €$ im Jahr 2009 um rund $5 \%$ über dem Durchschnitt von $387 €$. Der durchschnittliche Wohnungsaufwand pro $\mathrm{m}^{2}$ in Mietwohnungen lag dagegen mit $5,25 €$ unter dem Durchschnitt von 5,42€ (Statistik Austria 2010d). Angesichts der hohen Bodenpreise in Tirol ist überraschend, wie wenig die Wohnungskosten vom Durchschnittswert für Österreich abweichen. Dies kann auch nicht auf die Wohnbauförderung zurückgeführt werden. So hat etwa die Studie von Oberhuber, Amann und Bauernfeind (2005) ergeben, dass die Bewohner Tirols wie jene in Wien und Burgenland durch Zins- und Tilgungslasten im Rahmen geförderter Wohnbauten im Bundesländervergleich am stärksten belastet sind. Relativ zum Einkommen (siehe oben) sieht die Lage etwas ungünstiger aus. Im Durchschnitt jedenfalls geht es den Tirolern, was die Wohnungskosten betrifft, kaum schlechter als den Einwohnern in den übrigen Bundesländern. Diese Durchschnittsbetrachtung offenbart aber nicht, dass die Immobilienpreise in einigen zentralen Orten und vor allem im Zentralraum Innsbruck im österreichischen Vergleich sehr hoch sind.

Hohe Bodenpreise stellen für die Boden- und Wohnungspolitik eine Herausforderung dar, vor allem in verteilungspolitischer Hinsicht. In allokativer Hinsicht haben die Preise eine wichtige steuernde Funktion in Richtung flächensparender Siedlungsentwicklung. Ein hoher Bodenpreis ist Indikator für ein knappes Baulandangebot. Durch Bauland-

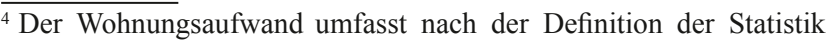
Austria die Wohnungsmiete bzw. die Annuität bei Eigentum sowie die Betriebskosten.
}

mobilisierung kann dieser Knappheit entgegengewirkt werden, ohne dass neue Flächen ausgewiesen werden müssen. Hohe Bodenpreise verschaffen auch Anreize zu dichterer Verbauung. Dies ist wünschenswert, solange das verdichtete Bauen den öffentlichen Anforderungen hinsichtlich Siedlungsqualität und Nutzerpräferenzen entspricht. Eine durch Staatsintervention herbeigeführte Absenkung der Bodenpreise ist bei sehr ungleicher Verteilung der Vermögen und Einkommen verteilungspolitisch wünschenswert. Es besteht dann aber immer auch die Gefahr eines verschwenderischen Umgangs mit dem Boden zu Lasten aller (ARGE ALP 2000: 13).

Festzuhalten bleibt, dass das Phänomen der Baulandzurückhaltung vermutlich kein allgemeines Problem der Tiroler Bodenmärkte ist, sondern eines, das lokal signifikante Wirkungen auf das Baulandangebot hat. Es stellt sich daher die prinzipielle Frage, ob mit der Einführung einer flächendeckenden, das heißt bundeslandweiten homogenen Widmungsabgabe den lokalen Bodenmarktproblemen begegnet werden kann. Wie sich im Folgenden noch erweisen wird, hätte die Widmungsabgabe für sich allein genommen gerade dort keine baulandmobilisierende Wirkung, wo sie am nötigsten wäre, nämlich in den zentralen und daher teuren Gunstlagen. Um dort Bauland zu mobilisieren, bedürfte es zusätzlicher Maßnahmen.

\section{Was ist ein Widmungsgewinn?}

Der Entwurf zur Novelle des Tiroler Raumordnungsgesetzes sah die Erhebung einer „Widmungsabgabe“ in jenen Fällen vor, in denen sich durch Umwidmung von Grundstücken im Vergleich zur vorherigen Widmung eine Steigerung des ortsüblichen Verkehrswertes ergibt. Die Widmungsabgabe sollte $10 \%$ der Steigerung des ortsüblichen Verkehrswertes betragen.

Eine Voraussetzung für ein umfassendes Verständnis der Wirkungen einer neuen Abgabe oder Steuer ist, Klarheit darüber zu schaffen, was das Wesen ihrer Bemessungsgrundlage ist. Im Zusammenhang mit der Widmungsabgabe ist $\mathrm{zu}$ fragen, was überhaupt unter einem Widmungsgewinn zu verstehen ist bzw. was die Gründe für das Entstehen von Widmungsgewinnen sind. Wie kann es sein, dass Umwidmungen von Freiland in Bauland Wert- und Preisveränderungen ermöglichen, die auf anderen Vermögensmärkten ihresgleichen suchen? Relative Preissprünge von über $1.000 \%$ sind üblich, manchmal sind sogar mehr als $10.000 \% \mathrm{zu}$ beobachten. Drei grundlegende Ursachen für derart kräftige Bodenwertsteigerungen sind Änderungen der Flächenwidmung, Änderungen der Bebauungsplanung und Verbesserungen des Angebots an öffentlichen Gütern durch Gebietskörperschaften und in deren Auftrag tätige (öffentliche) Unternehmen. Grundsätzlich hängt der Wert 
eines Grundstückes aber von mehreren Faktoren ab (vgl. in andersartiger Aufzählung beispielsweise Eekhoff 1987: 202 f. oder Bienert/Funk 2007: 188 f.). Die wichtigsten sind:

- die natürlichen Merkmale des Grundstücks,

- die natürlichen Umgebungsbedingungen (natürliche externe Effekte),

- eigene Aufwendungen des Grundstückseigentümers (z. B. Bodenverbesserungen),

- öffentliche Erschließungen (Straßen, Kanal und öffentliche Güter im weitesten Sinn),

- externe Effekte aus privater und privatwirtschaftlicher Aktivität,

- das Verhältnis von Angebot und Nachfrage am betreffenden Grundstücksteilmarkt und

- die grundstücksbezogene öffentliche Normsetzung (Flächenwidmung, Bebauungsplan).

Die Bedeutung jedes einzelnen dieser Faktoren hängt naturgemäß von der Nutzung des Grundstücks ab. Für Agrar- und Bauland sind nicht alle Faktoren gleichermaßen wertbeeinflussend. Daher ist die Beurteilung dessen, was genau unter einem Widmungsgewinn bzw. Umwidmungsgewinn zu verstehen ist bzw. was genau die Ursachen von Widmungsgewinnen sind, nicht trivial.

Sehen wir uns dazu die Gründe für die aus Umwidmungen resultierenden Preissprünge genauer an. Der Bodenwert richtet sich in erster Linie nach den Erträgen, die als Folge der Nutzung des Bodens erwartet werden. Im Bereich der Land- und Forstwirtschaft werden diese Erträge, sieht man von staatlichen Unterstützungsmaßnahmen ab, weitgehend auf (internationalen) Wettbewerbsmärkten bestimmt. Der aus den Erträgen ableitbare Bodenpreis für agrarisch genutzten Boden ist daher regional, je nach land- oder forstwirtschaftlicher Nutzung, weitgehend gleich. Preisunterschiede gibt es allenfalls aufgrund unterschiedlicher Bodenqualitäten und Transportkosten. Sie sind normalerweise gering im Vergleich zu den interregionalen Preisdifferenzen bei anders als landwirtschaftlich genutzten Böden.

Der Wert land- und forstwirtschaftlicher Grundstücke wird aber zusätzlich davon beeinflusst, ob erwartet wird, dass sie als Bauland ausgewiesen werden (Bauerwartungsland ${ }^{5}$ ) oder für eine andere höherwertige Nutzung zugelassen werden. Der Wert von Bauerwartungsland steigt in der Regel mit der Nähe zum vorhandenen Siedlungsraum und hängt entscheidend von den vorhandenen bzw. geplanten Änderungen der Lagequalitäten (Infrastrukturen und

\footnotetext{
${ }^{5}$ Unter dem Begriff „Bauerwartungsland“ wird hier im ökonomischen Sinne Grünland oder Freiland verstanden, für das eine Umwidmung in Bauland wahrscheinlich ist, auch wenn die Wahrscheinlichkeit sehr gering sein kann. Engere Definitionen finden sich in der ÖNORM B 1802 oder in der deutschen Wertverordnung (WertV) vgl. Bienert/Funk (2007: 203 f.)
}

andere öffentliche Güter im weitesten Sinne) ab. Unter der Annahme, dass zwei potenzielle Transaktionspartner bei einem Grundstück dessen baldige Umwidmung in Bauland erwarten, wird im Falle einer Transaktion der resultierende Kaufpreis für das Grundstück (als Bauerwartungsland) sehr nahe am Preis vergleichbaren Baulandes liegen. Ist auch nur einer der beiden Transaktionspartner weniger optimistisch hinsichtlich einer baldigen Umwidmung, so wird sich ein Kaufpreis in der Regel weiter unterhalb des Baulandpreises ergeben. Zudem können sich die Baulandpreise, im Gegensatz zu den Preisen für agrarisch genutzte Grundstücke, je nach regionaler Marktsituation sehr stark unterscheiden. Dabei hängt das Bodenpreisniveau von der Attraktivität der Lage ab, die ihrerseits bestimmt wird durch die Arbeitsmarktsituation, das Angebot an staatlicher Infrastruktur und anderen öffentlichen Gütern, die Umweltqualität etc. Das Verhältnis von Angebot und Nachfrage auf dem Markt der Grundstücke für höherwertige Nutzungen ist zum Teil durch relativ unelastische Nachfrage ${ }^{6}$ in Kombination mit Besonderheiten des Sektors (Wohnraumnachfrage; hohe Subventionierung, hohe Infrastrukturkosten) und zum Teil durch spezielle Anforderungen der Nutzung (Gewerbe- und Industrienutzungen, Standortoptimierung, Infrastruktur usw.) geprägt.

Der Hauptgrund für lokal auftretende Bodenpreisdifferenzen zwischen unterschiedlichen Nutzungen ist in der hoheitliche Flächenwidmung $\mathrm{zu}$ suchen, welche zu einer künstlichen, obgleich sozial- und umweltpolitisch gewünschten, Aufspaltung der Bodenmärkte führt. Auf freien Bodenmärkten gäbe es keine Preisdifferenzen zwischen unterschiedlich genutzten Grundstücken mit gleicher Standortgunst, weil sich immer nur jene Nutzung durchsetzt, für die die höchsten Erträge erwartet werden (Evans 2004: 80). Alle anderen Nutzungen würden verdrängt. Daher gäbe es auf freien Bodenmärkten kein Äquivalent zu Umwidmungsgewinnen.

Für das Verständnis der Umwidmungsgewinne sind also drei Aspekte von entscheidender Bedeutung. Zum ersten entscheidet im derzeit geltenden Rechtsrahmen ganz klar die Flächenwidmung über die zulässige Nutzung eines Grundstückes. Dadurch bewirkt sie eine Teilung der Bodenmärkte und diese Teilung bewirkt Preisdifferenzierung nach Nutzungsarten. Je strenger die Flächenwidmung ist, das

\footnotetext{
${ }^{6}$ Die Preiselastizität der Nachfrage ist das Verhältnis zwischen der relativen Veränderung der Nachfrage nach einem Gut und der relativen Veränderung des Preises, die die Nachfrageänderung verursacht. Kurz gesagt, sie zeigt, wie stark die Nachfrage reagiert, wenn sich der Preis um einen bestimmten Prozentsatz verändert. Ist die Reaktion „stark“, so spricht man von elastischer Nachfrage. Ist sie „schwach“, so spricht man von unelastischer Nachfrage. In der Regel ist die Nachfrage nach elementaren Gütern des Lebens eher preisunelastisch. Dies gilt beispielsweise für Wohnen, Gesundheit, Ernährung, Energie, Verkehr. In diesen Bereichen liegt die Preiselastizität zumeist im Bereich von etwa $-0,3$ bis $-0,5$.
} 
heißt, je restriktiver aufwertende Umwidmungen erfolgen, desto höher sind unter sonst gleichen Bedingungen die Nutzungspreisdifferenzen und desto höher sind die potenziellen Umwidmungsgewinne.

Die mit Umwidmungen verbundenen Bodenwertsteigerungen hängen darüber hinaus vom Verhältnis von Angebot und Nachfrage nach bestimmten Nutzungen ab. Eine Umwidmung, etwa von Freiland in Bauland, hebt nur eine vorher bestehende Beschränkung auf und lässt damit zu, dass sich Angebot und Nachfrage nach Bauland nun frei von lokal differenzierter Staatsintervention entfalten können. Sowohl Angebot als auch Nachfrage nach Bauland waren immer latent vorhanden, was sich vor allem in den Preisen für Bauerwartungsland widerspiegelt. Diese liegen regelmäßig über den Preisen von Freiland, obwohl noch keine entsprechende Widmung als Bauland besteht.

Zusätzlich zur Flächenwidmung und der Marktsituation ist auch die Bebauungsplanung entscheidend für die Höhe der Umwidmungsgewinne. Die Bodenerträge werden wesentlich beeinflusst von der zulässigen Baufläche und der zulässigen Bauhöhe. In welchem Ausmaß diese Faktoren jeweils wirken, ist kaum systematisch erforscht. ${ }^{7}$

\section{Die Tiroler Widmungsabgabe im Lichte der bodenökonomischen und finanzwissenschaftlichen Diskussion}

In den folgenden sieben Unterkapiteln werden die wichtigsten Wirkungen und Probleme einer Widmungsabgabe aus bodenökonomischer und finanzwissenschaftlicher Sicht qualitativ untersucht.

\subsection{Abschöpfung von staatlich induzierten Grundstückswerterhöhungen}

Die mit Umwidmungen verbundenen Ertragsänderungen und Wertveränderungen des Bodens sind seit langem im Blick der bodenpolitischen Diskussion. In Deutschland wurde seit den 1960er Jahren und in der Schweiz seit Anfang der 1970er Jahre über die Abschöpfung von Wertsteigerungen von Grundflächen als Folge von Änderungen der Flächenwidmung, Änderung von Bauordnungsnormen und/oder von öffentlichen Infrastrukturinvestitionen diskutiert (z. B. Markstein 2004; Junker 2010). In Österreich gab es im Jahr 2002 eine Fachenquete zum Thema „Zeitgemäße Planungsinstrumente - Modell Planwertabgabe“ (Lechner

\footnotetext{
${ }^{7}$ Ausnahmen bilden die Arbeiten von Glaeser und Gyourko (2003), Salvi (2004) und Wieser (2008), welche mit Hilfe von hedonischen Bodenpreismodellen die Wirkungen von Bauordnung und Flächenwidmung auf die Bodenpreise untersuchen.
}

2006). Eine gesetzliche Verankerung wie in der Schweiz ${ }^{8}$ seit 1976 oder zumindest konkrete Vorschläge, wie sie in Deutschland ${ }^{9}$ vorliegen, gibt es in Österreich bislang noch nicht. Das Tiroler Modell stellt hier einen ersten legistischen Versuch dar.

Die Abschöpfung von Grundstückswerterhöhungen als Folge von Umwidmungen und Erschließungsmaßnahmen wird in der wissenschaftlichen Literatur als „Plan(ungs)wertausgleich" bezeichnet, herbeigeführt durch Erhebung einer „Planwertabgabe“. Ihre Höhe bemisst sich entweder an den tatsächlichen Kosten der jeweiligen staatlichen Maßnahmen (sogenannte Beitragslösung) oder an den Wertzuwächsen der einzelnen Grundstücke (sogenannte Wertlösung) (vgl. Lechner 2006). Die in der Novelle zum Tiroler Raumordnungsgesetz vorgeschlagene Widmungsabgabe stellt eine reine Wertlösung dar, daher wird hier kurz auf die wissenschaftliche Diskussion zur Wertlösung eingegangen.

Das ursprüngliche Ziel einer Abschöpfung von staatsinterventionsbedingten Grundstückswertserhöhung ist, einen sachgerechten Ausgleich zwischen den Interessen des Grundeigentümers und jenen der Allgemeinheit herbeizuführen. Ohne einen solchen Ausgleich würde eine Gruppe von Grundeigentümern leistungslos von den Maßnahmen profitieren, während eventuell anfallende Kosten (von Infrastrukturmaßnahmen) der Allgemeinheit teilweise aufgebürdet würden. Entstünde umgekehrt den Eigentümern infrastruktur- und widmungsbenachteiligter Grundstücke ein Wertverlust (z. B. weil ein Grundstück durch eine neu angelegte Straße geteilt wird oder weil auf eine höherwertige Nutzung des Grundstückes verzichtet werden muss), dann wäre aus Gründen der Symmetrie der Schaden von der Allgemeinheit (der öffentlichen Hand) im Wege einer Entschädigung abzugelten. Aus steuertheoretischer Sicht entspricht eine Abschöpfung von staatsinterventionsbedingten Mehrwerten dem (marktwirtschaftlichen) Äquivalenzprinzip (Eekhoff 1987: 224; vgl. Lechner 2006).

Neben der steuertheoretischen Rechtfertigung von „Planwertausgleichen“ interessieren vor allem die allokativen und distributiven Wirkungen eines solchen Instruments am

\footnotetext{
${ }^{8}$ In der Schweiz haben die Kantone den bundesgesetzlichen Auftrag, einen angemessenen Ausgleich von Vor- und Nachteilen sicherzustellen. Zum Teil wird dies durch Grundstücksgewinnsteuern erreicht. Diese beziehen sich aber auf sämtliche Mehrwerte, nicht nur die staatsinterventionsbedingten. Nur zwei Kantone, Basel-Stadt und Neuenburg, haben spezifische Abschöpfungsinstrumente bzw. Ausgleichsmechanismen eingeführt (vgl. Markstein 2004: 99 f.). Waber (2010) berichtet zur aktuellen Diskussion in der Schweiz.

${ }^{9}$ Gegen Ende der 1990er Jahre haben die Bundestagsfraktionen von SPD und Bündnis 90/Die Grünen den Versuch unternommen, den Regierungsentwurf des BauROG 1998 um Regelungen zur Einführung eines „Planwertausgleichs“ zu ergänzen. „Im Rahmen des Planwertausgleichs sollten $70 \%$ der planungsbedingten Bodenwerterhöhung als Abgabe von den planungsbegünstigten Grundstückseigentümern erhoben werden (Junker 2010: Seite 33).“
} 
Bodenmarkt. Unter allokativer Wirkung versteht man die Auswirkungen einer Umwidmung auf die Grundstücksnutzung, also deren kausale Veränderung. Dabei geht es um die Frage der volkswirtschaftlichen Effizienz der neuen Nutzungsmöglichkeit, das heißt, inwieweit die Wohlfahrt netto zunimmt. Als distributiv werden die Wirkungen auf die persönliche und gruppenspezifische (auch räumliche) Einkommens- und Vermögensverteilung bezeichnet. Die allokativen und distributiven Wirkungen sind allerdings nicht ganz einfach zu prognostizieren, weil stark kontextabhängig (Eekhoff 1987: 249 ff.; Evans 2004: 103 ff. und 109). Es könnte sein, dass die Abschöpfung von staatsinterventionsbedingten Änderungen von Grundstückswerten dazu führt, dass die Eigentümer nach der Umwidmung das Grundstück verkaufen und die der Abgabe entsprechende Summe auf den Verkaufspreis aufschlagen wollen. Inwieweit dies gelingt bzw. zutrifft, hängt von der Marktlage und von den Erwartungen über die zukünftigen Marktentwicklungen ab. In Regionen mit tendenziell unelastischer Baulandnachfrage könnte eine Überwälzung gelingen, wodurch die Baulandpreise dort zusätzlich steigen würden. Abgesehen davon ist nicht unbedingt damit zu rechnen, dass durch Einsatz dieses bodenpolitischen Instruments auch die Bodenmobilität steigt. Die Verkaufsbereitschaft hängt bei rationalen Akteuren allein von der erwarteten Entwicklung der Bodenpreise nach Umwidmung und der Opportunitätskosten der Bodenhortung (Zinsentgang durch fortgesetzte Bindung von Kapital) ab. Dieses Kalkül wird durch einen „Planwertausgleich" nicht außer Kraft gesetzt. Allerdings vermindert diese Abgabe die Gesamtrentabilität der Bodenhortung, es sei denn, sie kann im Zuge der Veräußerung vollständig auf den Grundstückskäufer überwälzt werden.

Die Stärke der Verkaufs- und Bebauungsanreize im Zusammenhang mit der Einführung einer Widmungsabgabe hängt von deren konkreter Ausgestaltung ab. Für die vorgeschlagene Widmungsabgabe in Tirol stellen sich im Lichte der angeführten Argumente einige Fragen, die nachfolgend untersucht werden.

\subsection{Ermittlung von Wertsteigerungen}

Der Entwurf zur Novelle des Tiroler Raumordnungsgesetzes sah für die Widmungsabgabe vor (TROG-Novelle 2010), dass

1. die gesamten Wertsteigerungen als Folge von Umwidmungen als Bemessungsgrundlage dienen,

2. nicht durch Transaktionen objektivierte Grundstückswerte (Marktwerte), sondern Schätzwerte zugrunde gelegt werden und

3. dabei die durch Gutachter festgestellten ortsüblichen Schätzwerte vor und nach Widmung zur Anwendung kommen.
Ad (1): Die vorgeschlagene Widmungsabgabe stellt einen sehr pragmatischen Ansatz dar. Nach den Ursachen der umwidmungsbedingten Wertsteigerungen wird gar nicht gefragt. Theoretisch ist nämlich vorstellbar, dass Wertsteigerungen nach Umwidmung nicht nur auf staatliche Interventionen (z. B. Bereitstellung von Infrastruktur) zurückführbar sind. Es könnten auch private Investitionen (z. B. Maßnahmen zur Bodenverbesserung, wie etwa Hangverflachungen vor Bebauung) zur Wertsteigerung beigetragen haben. Die Widmungsabgabe ginge also über den Charakter eines reinen „Plan(ungs)wertausgleich“ hinaus. Die Problematik, dass das Zurechnungsproblem (worauf genau sind die Wertsteigerungen zurückzuführen?) zu Lasten der privaten Eigentümer entschieden wird, wäre allerdings durch die geringe Höhe der Abgabe (nur $10 \%$ der Widmungsgewinne) gemildert gewesen. Zudem dürfte in den meisten Fällen ein Großteil der Grundstückswertsteigerungen auf das Vorhandensein oder die geplante Bereitstellung nutzungskomplementärer öffentlicher Güter zurückzuführen sein.

Ad (2) und (3): Die im Entwurf vorgeschlagene Anwendung von ortsüblichen Schätzwerten nach Wirksamwerden der Widmung anstelle der Anwendung des Realisationsprinzips wirft weitere Fragen auf. Zweck des Vorschlages dürfte neben der fiskalischen Funktion auch eine Erhöhung der Baulandmobilität gewesen sein (dazu finden sich in den Erläuterungen zur Novelle allerdings keine näheren Ausführungen). In der Tat wäre zu befürchten, dass bei Anwendung des Realisationsprinzips, das heißt Fälligkeit der Widmungsabgabe erst bei Übertragung bzw. Transaktion eines Grundstückes, die Baulandmobilität nicht steigen würde, sondern sogar verringert werden könnte. Im Hinblick auf die Bodenmobilität ist die vorgeschlagene Konzeption daher zumindest nicht inferior.

Eine Schwäche in der Konzeption der Widmungsabgabe hätte sich wohl auch in der Anwendung der Schätzwerte offenbart. Was wäre passiert, wenn die Grundeigentümer mit den ermittelten ortsüblichen Schätzwerten nicht einverstanden gewesen wären und diese rechtlich angefochten hätten? Dieses Problem wäre bei Anwendung des Realisationsprinzips nicht aufgetreten, da ja in dem Fall objektive Transaktionspreise zugrunde gelegen hätten. $\mathrm{Zu}$ bedenken ist allerdings, dass auch Transaktionspreise durch Nebenabsprachen, kompensierende Maßnahmen und letztlich auch durch „steuerschonende“ Konstruktionen verzerrt sein können.

\subsection{Die Frage der baulandmobilisierenden Wirkung der Widmungsabgabe}

Die Baulandhortung wird weithin als ein zentrales Problem der Tiroler Bodenpolitik angesehen. Allerdings hat Baulandhortung nicht immer nur negative Auswirkungen auf die Wohlfahrt einer Gesellschaft. Bedenkenswerte Gründe 
der Hortung sind der frühzeitige Erwerb von Boden für die Nachkommen, um spätere Grundpreissteigerungen abzufangen (Versicherungsmotiv), oder der frühzeitige Erwerb von Boden durch Bauträger, um später ein Bauvorhaben zu realisieren (marktgesteuertes Bebauungsmotiv).

Problematisch ist die Baulandhortung (in Tirol) unter folgenden Bedingungen. Viele Grundstücke sind durch Hypotheken belastet, die einem anderen als dem Bauzweck dienten. Dies verhindert die politische Durchsetzbarkeit von Rückwidmungen, die bei Vorliegen lokal großer Baulandüberhänge raumordnungspolitisch oft sinnvoll wären. In manchen Regionen führen Baulandhortungen zur Ausuferung des Siedlungsgebietes und zu Planungsunsicherheit bei den Gemeinden (Standorte und Dimensionierung von öffentlichen Einrichtungen, zeitgleiches Auftreten von Kapazitätsengpässen und Unterauslastungen bei öffentlichen Einrichtungen). Vor allem in den zentralen Regionen hat die Baulandhortung auch preistreibende Wirkung. Hohe Bodenpreise erschweren dort den (subventionierten) Wohnbau für mittlere und untere soziale Schichten. Dies ist nicht nur ein soziales oder verteilungspolitisches Problem. Es kann dadurch auch zu einer dauerhaften Unterauslastung bereits vorhandener öffentlicher Infrastruktur kommen. Durch die Verdrängung der unteren sozialen Schichten in Randlagen können darüber hinaus in diesen Lagen negative Effekte entstehen.

Die Diskussion um den „Planwertausgleich“ hat bereits gezeigt, dass eine einmalige Abgabe das Kalkül spekulativ denkender Grundstückseigentümer in keiner Weise verändert (vgl. Kap. 4.1). Solange die erwarteten prozentuellen Bodenpreissteigerungen über dem Zinssatz alternativer Veranlagungen liegen, werden Grundstücke gehortet, denn die erwarteten Preissteigerungen überkompensieren die entgangenen Zinsgewinne (Opportunitätskosten der Baulandhortung). Eine baulandmobilisierende Wirkung einer Widmungsabgabe ist nur dann zu erwarten, wenn die von den Bodeneigentümern erwarteten Preissteigerungen bei Bauland unter der risikoadäquaten Kapitalverzinsung liegen. Dies wird üblicherweise nur in strukturschwachen Randregionen der Fall sein. Daher besteht in solchen Regionen die Gefahr, dass durch die Widmungsabgabe die Zersiedelung stärker und schneller vorangetrieben wird, weil dort aus Gründen der Einkommensgenerierung schneller verkauft wird. Als Instrument der Baulandmobilisierung ist die Widmungsabgabe gerade in jenen Regionen nicht geeignet, in denen am meisten über die Baulandhortung geklagt wird, nämlich in Regionen mit hohem Nachfragedruck und hohen Bodenpreisen, das heißt in Regionen, wo weiterhin mit tendenziell stärker steigenden Bodenpreisen gerechnet werden muss.

\subsection{Direkte Verteilungswirkungen}

Die Widmungsabgabe hätte unbestreitbar zu einer Umverteilung von den (privaten) Grundeigentümern, seien es jene vor dem Verkauf von Grundstücken oder die neuen Eigentümer nach einem Verkauf, zum öffentlichen Sektor geführt. Dies war auch ein erklärtes Ziel dieser Maßnahme. Empfänger der Erträge aus der Widmungsabgabe wären das Land und der Tiroler Bodenfonds gewesen. Dieser hätte, wie in den Erläuterungen zum Gesetzesentwurf ausgeführt wurde, die zusätzlichen Mittel aus der Widmungsabgabe zum Zweck der Erfüllung seiner Aufgaben einzusetzen gehabt. Vor allem sollte der Bodenfonds in die Lage versetzt werden, vermehrt Grundstücke zu erwerben und diese zu ,angemessenen“ Preisen im Rahmen der Fondszwecke weiterzuveräußern. Einen Schwerpunkt bildete dabei die Schaffung von Wohnraum zu „leistbaren, sozial verträglichen" Preisen. Die Argumentation in den Erläuterungen lässt darauf schließen, dass der Gesetzgeber in der vorgesehenen Widmungsabgabe in erster Linie ein Instrument der Umverteilung gesehen hat, indem durch teilweise Abschöpfung von Umwidmungsgewinnen der soziale Wohnbau gestärkt bzw. Anspruchsberechtigte im sozialen Wohnbau unterstützt hätten werden sollen.

Demgemäß wäre in Zukunft also mehr an relativ günstigem Wohnraum zur Verfügung gestanden, vor allem dann, wenn der Bodenfonds seine gestärkte finanzielle Basis genutzt hätte, um seinerseits Grundstücke unter Marktpreisen bereitzustellen. In räumlicher Hinsicht wäre es zu einer Umverteilung zu neuen Wohnungsinhabern in jenen Regionen bzw. zu jenen Gemeinden gekommen, in denen der Bodenfonds am stärksten aktiv gewesen wäre. Widmungsgewinne entstehen in allen Gemeinden, in denen gewidmet wird. Die höchsten Widmungsgewinne entstehen aber dort, wo die Bodenpreise hoch sind und dies ist in der Regel in den reicheren Gemeinden der Fall. Der Bodenfonds hätte erheblich zwischen den Gemeinden umverteilen können. Eine Abschwächung der räumlichen Verteilungswirkungen hätte sich aber daraus ergeben, dass Umwidmungsgewinne auch bei der Vertragsraumordnung ${ }^{10}$ zum Tragen gekommen wären, da auch für den geförderten Wohnbau gewidmete Flächen von der Widmungsabgabe betroffen gewesen wären.

Aus verteilungspolitischer Sicht ist auch von Interesse, welche Marktseite letztendlich die Abgabenlast zu tragen gehabt hätte. Diese Frage kann mangels entsprechender

\footnotetext{
${ }^{10}$ Unter Vertragsraumordnung versteht man die Kombination von Flächenwidmung mit privatrechtlichen Verträgen zwischen Grundeigentümern und Gemeinde zu dem Zweck, die politisch beabsichtigte Nutzung der einzelnen Grundstücke, wie sie in der Flächenwidmung zum Ausdruck kommt und durch sie allein aber voraussichtlich nicht oder nur teilweise erreichbar ist, tatsächlich zu realisieren (ähnlich Amt der Niederösterreichischen Landesregierung 2007: 4).
} 
Daten und eines darauf aufbauenden Modells des Bodenmarktes hier nur qualitativ beantwortet werden. Die finanzwissenschaftliche Abgabeninzidenzanalyse geht davon aus, dass jeweils jene Marktseite den größeren Teil der Abgabenlast trägt, die weniger Alternativen hat. Im Wohnungssektor wird normalerweise davon ausgegangen, dass mittelfristig die Nachfrage auf Preisveränderungen unelastischer reagiert als das Angebot. Damit hätten vor allem die Nachfrager (Mieter, Eigentumswohnungskäufer) den Hauptteil der Abgabenlast zu tragen gehabt. Die Grundstückseigentümer bzw. -verkäufer hätten vermutlich einen Großteil der Widmungsabgabe über höhere Bodenpreise auf die künftigen Nutzer abwälzen können. Im Bereich der Gewerbe- und Industriebetriebe wäre das Ergebnis ähnlich gewesen. Vor allem größere Betriebe wären aber wahrscheinlich besser in der Lage gewesen, eine vollständige Überwälzung abzuwehren. Insgesamt wäre die Belastung aufgrund des moderaten Abgabensatzes von $10 \%$ aber nicht dramatisch gewesen. Selbst wenn es den Grundstückeigentümern bzw. -verkäufern gelungen wäre, die volle Abgabenlast auf die Nutzer abzuwälzen, wären die Baulandpreise ceteris paribus um nicht mehr als $10 \%$ angestiegen. Geht man von einem lokalen durchschnittlichen Anteil der Grundkosten an den Gesamtbaukosten im Geschossbau von $20 \%$ aus, so wären die Gesamtbaukosten durch die volle Überwälzung um nicht mehr als $2 \%$ gestiegen. Und dies wäre ein Extremfall gewesen. Im Bereich der Eigenheime wäre der Prozentsatz aufgrund des höheren Grundkostenanteils gröBer gewesen, was allerdings sozial- und wohnungspolitisch wenig bedenklich erscheint.

\subsection{Die Stärkung des Bodenfonds - allokative Aspekte}

Der Tiroler Bodenfonds ist eine Körperschaft mit eigener Rechtspersönlichkeit und gemeinnützig ausgerichtet. Der Fonds verfügte im Jahr 2005 über ein Eigenkapital von rund 1,6 Mio. $€$ bei einer Bilanzsumme in Höhe von 24,4 Mio. €. Die Eigenkapitalquote betrug demnach 6,6\% (Landesrechnungshof Tirol 2006: 29). Das Aufkommen der Widmungsabgabe hätte, wäre sie im Jahr 2008 bereits eingeführt gewesen, allein in diesem Jahr jedenfalls mehr als 10 Mio. $€$ betragen. ${ }^{11}$ Durch die Zuweisungen der Erträge aus der Widmungsabgabe wäre daher das Eigenkapital des Bodenfonds schon im ersten Jahr um voraussichtlich mindestens den Faktor 6 höher gelegen und hätte sich in den Folgejahren noch erheblich gesteigert. Binnen zehn Jahren hätte das Eigenkapital theoretisch auf 100 Mio. $€$ anwachsen und, bei Aufrechterhaltung der ursprünglichen Eigenkapitalquote, ein Bilanzsumme von 1,5 Mrd. € generieren können. Selbst wenn man eine Eigenkapitalquote von $25 \%$

\footnotetext{
${ }^{11}$ Persönlichen Gesprächen mit Tiroler Bodenmarktexperten zufolge hätte das Aufkommen sogar 20 Mio. $€$ oder mehr betragen.
}

unterstellt, eine Quote wie sie beim Landeskulturfonds vorlag (Landesrechnungshof Tirol 2006: 29), wäre binnen zehn Jahren ein Bilanzsumme von 400 Mio. $€$ vorstellbar gewesen. Allerdings darf nicht übersehen werden, dass der Fonds ja Grundstücke für den sozialen Wohnbau günstig abgeben sollte. Wenn er Bauland zu (von ihm zusätzlich beeinflussten) Marktpreisen erworben und dieses günstiger abgegeben hätte, wären seine Eigenkapitalbasis und damit verbunden auch das Bilanzsumme natürlich langsamer angewachsen.

In Stromgrößen gerechnet, hätte der Bodenfonds durch Aufnahme von Fremdkapital, je nach Eigenkapitalquote, jährlich Grundstückskäufe in einer Größenordnung durchaus von 60 bis 80 Mio. $€$ durchführen können. Derzeit beträgt der geschätzte Geldwert an neu gewidmetem Bauland etwa 120 Mio. $€$ pro Jahr, die entsprechenden Freilandwerte lagen bei weniger als 10 Mio. $€$ (vgl. TROG-Novelle, Erläuternde Bemerkungen 2010). Die Grundstückskäufe des Bodenfonds hätten also in kürzester Zeit eine Größenordung erreichen können, die weit über dem jährlichen Geldwert des umgewidmeten Freilandes gelegen wären. Wenn man zudem berücksichtigt, dass in $90 \%$ der Gemeinden derzeit Baulandüberhänge bestehen, muss angenommen werden, dass der Bodenfonds seine Aktivitätsschwerpunkte vom bisher im Mittelpunkt stehenden Freilandkauf mit späterer Umwidmung verstärkt in Richtung des Kaufs von bereits gewidmetem Bauland geändert hätte. Mit der Stärkung der finanziellen Basis des Fonds in vielen Fällen verbunden gewesen wäre ein hohes $\mathrm{Ma}$ an Verhandlungsmacht gegenüber den Grundeigentümern. In manchen Fällen wären aber auch zusätzlich die Bodenpreise dadurch gestiegen, dass der Bodenfonds bei bestimmten Grundstücken als zusätzlicher Konkurrent neben privaten Nachfragern aufgetreten wäre.

Insgesamt wäre der Bodenfonds durch die Zuweisung der Mittel aus der Widmungsabgabe zum wichtigsten Akteur am Tiroler Bodenmarkt avanciert. In allokativer Hinsicht hätte er je nach regionaler Bodenmarktlage Nachfragemacht ausspielen können oder wäre als zusätzlicher kaufkraftstarker Nachfrager tätig geworden. Er hätte in, aus raumordnungspolitischer Sicht, strategisch wichtigen Lagen baulandmobilisierend agieren können und durch seine Aktivitäten darüber hinaus wohl auch die räumliche Verteilung von Wohnbauförderungsgeldern des Landes beeinflusst. Die allokativen Wirkungen der Aktivitäten des Fonds am Bodenmarkt wären vermutlich beträchtlich gewesen.

\subsection{Die Widmungsabgabe im Verbund mit anderen steuerpolitischen Maßnahmen und Instrumenten}

In den Erläuterungen zum Entwurf der Novelle wurde die Widmungsabgabe vor allem aus kompetenzrechtlicher und steuerpolitischer Sicht diskutiert. Es ging dabei darum, ob die Einführung einer Widmungsabgabe an eine bundesgesetzliche Ermächtigung geknüpft ist. Dazu wurde ausgeführt, 
dass die Widmungsabgabe weder der als gemeinschaftliche Bundesabgabe konzipierten Bodenwertabgabe noch der als bundesgesetzlich geregelten Gemeindeabgabe Grundsteuer gleichartig gewesen wäre. Die Bodenwertabgabe wird periodisch wiederkehrend für unbebaute Grundstücke erhoben, wobei als Bemessungsgrundlage der Einheitswert bzw. ein vom Einheitswert abgeleiteter Messbetrag dient. Auch die Grundsteuer wird periodisch auf der Grundlage des Einheitswertes erhoben. Keine der beiden Steuern stellt einen Zusammenhang mit dem Widmungsgewinn her.

Aus volkswirtschaftlicher bzw. raumplanerischer Sicht stellt sich die Frage hinsichtlich der Beziehung zwischen Widmungsabgabe und anderen steuerpolitischen und raumplanerischen Maßnahmen in anderer Weise. Es geht um die Wechselwirkungen bzw. um die allokativen und distributiven Wirkungen im Gesamtkonzert der Maßnahmen. Weder die Bodenwertabgabe noch die Grundsteuer haben in Österreich merkliche allokative oder distributive Wirkungen. Die Bodenwertabgabe wäre von ihrer Konzeption her gesehen ein ideales Mobilisierungsinstrument. Einzige Möglichkeit der Abgabe zu entgehen ist die widmungskonforme Bebauung unbebauter Baulandgrundstücke. Sie wäre daher bestens geeignet, die Mobilität des Baulandmarktes zu fördern. Allerdings ist derzeit die Belastung durch die Bodenwertabgabe derartig gering, dass sie keine bodenmobilisierende Wirkung erzielt. Der Grundsteuer wiederum fehlt auch aufgrund von Konzeption und geringem Steuersatz die bodenmobilisierende Wirkung.

Die stärkste allokative Wirkung am Bodenmarkt hat gegenwärtig die Regelung zu Veräußerungsgeschäften (Spekulationsgeschäften) im Einkommensteuerrecht. Die Regelung zur Spekulationsfrist im Rahmen der Einkommensteuer trifft den Grundeigentümer in dem Moment, in dem er bereit ist, die Bodenhortung aufzugeben (Realisationsprinzip). Spekulationsgeschäfte im Sinne des Einkommensteuerrechts sind Veräußerungsgeschäfte, bei denen der Zeitraum zwischen Anschaffung und Veräußerung bei Grundstücken nicht mehr als zehn Jahre beträgt. Besteuert wird hier der Gewinn aus Anschaffung und Veräußerung ${ }^{12}$. Diese Regelung hat daher stark negative Auswirkungen auf die Bodenmobilität. Auch die Grunderwerbsteuer, die in Österreich im internationalen Vergleich recht hoch ist (es gibt allerdings unter bestimmten Bedingungen ermäßigte Sätze) und als Teil der Transaktionskosten bei Grundstückstransaktionen anfällt, wirkt sich negativ auf die Bodenmobilität aus. Eine systematische Diskussion des eventuellen Einsatzes der beiden genannten Abgaben und der Einkom-

\footnotetext{
${ }^{12}$ Bei Eigentumswohnungen und Eigenheimen, die der Veräußerer seit der Anschaffung mindestens zwei Jahre durchgehend als Hauptwohnsitz benutzt hatte, ist die Spekulationsfrist auf zwei Jahre reduziert. Unentgeltliche Erwerbe werden durchgerechnet. Eine $10 \%$-Minderung ab dem fünften Jahr gilt bei unbebauten Grundstücken.
}

mensteuerregelung für Zwecke der Baulandmobilisierung gibt es in Österreich bislang nicht.

\subsection{Die Widmungsabgabe im Verbund mit anderen raumplanerischen Maßnahmen und Instrumenten}

Neben den Wirkungen der Widmungsabgabe im Kontext der genannten Abgaben gilt es auch, die Wechselwirkungen mit Maßnahmen und Instrumenten der Raumplanung zu diskutieren. Hier geht es beispielsweise um die Anreizwirkungen der Widmungsabgabe im Bereich der widmenden Behörden und um die Auswirkungen der Widmungsabgabe auf die Vertragsraumordnung. So etwa ist prüfenswert, ob nicht der derzeitige gezielt herbeigeführte privatwirtschaftlich geprägte Status des Tiroler Bodenfonds durch den starken Zufluss von Mitteln aus Erträgen aus einer Landesabgabe zu stark in die Nähe der Handlungsbedingungen eines öffentlichen Akteurs gerückt würde, so dass die Legalität des Abschlusses der privatrechtlichen Verträge im Rahmen der Vertragsraumordnung eventuell verloren gegangen wäre.

Gegenwärtig ist Flächenwidmung Gemeindesache, wogegen die Netto-Erträge aus der Widmungsabgabe an den Tiroler Bodenfonds hätten fließen sollen. Anreiztheoretisch betrachtet wäre die institutionelle Trennung zwischen Widmungszuständigkeit und den Einnahmen aus der Widmungsabgabe zu begrüßen gewesen. Dadurch wäre einerseits vermieden worden, dass die Gemeinden zu starke Anreize für Umwidmungen erhalten, wobei sich diese Anreize aber je nach Situation stark unterscheiden hätten können. Andererseits erwachsen den Gemeinden im Zusammenhang mit Baulandwidmungen regelmäßig hohe Aufwendungen. Daher wäre zu überlegen gewesen, zumindest einen Teil des Aufkommens aus der Widmungsabgabe den Gemeinden zuzuführen. Diese hätten dadurch beispielsweise auch befähigt werden können, unabhängig von den Aktivitäten des Bodenfonds kommunale Bodenbevorratung $\mathrm{zu}$ betreiben. Natürlich hätte es für diesen Fall entsprechende Regelungen geben müssen, die verhindern, dass sich Gemeinden und Bodenfonds als Konkurrenten am Bodenmarkt gegenseitig behindern. Eine Möglichkeit wäre gewesen, den Gemeinden eine Vorrangstellung bzw. eine Option in gewissem Umfang einzuräumen. Wenn sie die Option, in einem bestimmten Zeitraum Bodenvorräte anzukaufen, nicht oder nicht im vorgesehenen Ausmaß ausgeübt hätten, hätte der Bodenfonds freie Hand bekommen können. Eine solche Option wäre flexibler als die im Entwurf der Novelle vorgesehene Regelung.

Letztlich ist auch zu hinterfragen, ob die Stärkung der Stellung des Bodenfonds im prognostizierten Ausmaß (siehe oben) volkswirtschaftlich nicht auch negative Wirkungen hätte haben können. Es wäre hier zu einer Machtkonzentration gekommen, deren Folgen nicht leicht vorherzusehen gewesen wären. Ein wichtiger Aspekt in diesem Zusam- 
menhang ist ein psychologischer. Die Widmungsabgabe hätte den Effekt haben können, dass Grundeigentümer in ihr eine Abgeltung für öffentlich finanzierte bodenwertsteigernde Maßnahmen sehen. Dadurch hätte in Zukunft die Verhandlungsbereitschaft bzw. der „Goodwill“ der Grundeigentümer in Verhandlungen etwa mit dem Bodenfonds in Grundstücksfragen beeinträchtigt sein können. Neuere Forschungsergebnisse im Bereich der Verhaltensforschung und experimentellen Ökonomie verweisen auf starke Verhaltensänderungen der Akteure in Folge der Einführung von Sanktionsinstrumenten wie Beitragserhöhungen oder Steuern. ${ }^{13}$ Es ist nicht auszuschließen, dass sich hier geänderte Einstellungen in Bezug auf zukünftige vertragsrechtliche Regelungen im Rahmen der Raumplanung ergeben hätten.

\section{Fazit}

Der von der Tiroler Landesregierung im Sommer 2010 vorgelegte Entwurf zur Novelle des Tiroler Raumordungsgesetzes sah die Einführung einer Widmungsabgabe in Höhe von $10 \%$ des Widmungsgewinnes bei aufwertenden Umwidmungen vor. Dies hätte vor allem Widmungen von Freiland in Bauland oder in Sonderflächen betroffen. Das NettoAufkommen aus der Widmungsabgabe sollte dem Tiroler Bodenfonds zufließen, welcher dazu angehalten gewesen wäre, die finanziellen Mittel vor allem zum Ankauf zusätzlicher Grundflächen zur Unterstützung des sozialen Wohnbaus einzusetzen.

Hauptziel der Widmungsabgabe wäre eine Umverteilung der Widmungsgewinne von privaten Grundeigentümern bzw. Grundstücksnutzern zur öffentlichen Hand und in weiterer Folge zu den Anspruchsberechtigten im sozialen Wohnbau gewesen. Diese Umverteilung erschiene aus finanzwissenschaftlicher Sicht unbedenklich, weil normalerweise ein Großteil der widmungsbedingten Bodenmehrwerte durch Leistungen der öffentlichen Hand ohne volle Kostendeckung durch die Nutzer mitverursacht ist. Insofern entspricht die Idee einer solchen Umverteilung dem (marktwirtschaftlichen) Äquivalenzprinzip der ökonomischen Steuerlehre.

Die Stellung des Tiroler Bodenfonds am Tiroler Bodenmarkt wäre durch die Zuweisung der Mittel aus der Widmungsabgabe erheblich gestärkt worden. Die Aktivitäten des Bodenfonds hätten Umverteilungen in personeller und räumlicher Hinsicht bewirkt. In personeller Hinsicht hätten vor allem die Anspruchsberechtigten im sozialen Wohnbau profitiert. In räumlicher Hinsicht wäre es aufgrund der Tatsache, dass Widmungsgewinne überall dort entstehen, wo gewidmet wird, der Bodenfonds aber nicht überall tätig

\footnotetext{
${ }^{13}$ Einen guten Überblick zu den neueren Erkenntnissen der ökonomischen experimentellen Verhaltensforschung gibt Traurig (2009).
}

gewesen wäre, zu einer entsprechenden Umverteilung zwischen Tiroler Gemeinden gekommen. Aus allokativer Sicht hätte die Zuweisung der Mittel an den Bodenfonds und nicht an die Gemeinden wahrscheinlich Vorteile gehabt. Die institutionelle Trennung von Widmungsentscheidung und Abgabenerlös verhindert allokative Verzerrungen.

Die hier vorgelegte qualitative finanzwissenschaftliche Inzidenzanalyse hat ergeben, dass die Abgabenlast mit hoher Wahrscheinlichkeit zu einem größeren Teil nicht auf die privaten Grundstücksverkäufer entfallen wäre, sondern auf die privaten zukünftigen Nutzer (Grundstückseigentümer, die nicht verkaufen und selber nutzen wollen, Mieter, Pächter, Eigentumswohnungskäufer bzw. -nutzer etc.). Angesichts des moderaten Abgabensatzes von $10 \%$ des Widmungsgewinnes wären die Überwälzungswirkungen (Preissteigerungen) allerdings nicht dramatisch gewesen. Die Überwälzung der Abgabe auf die zukünftigen Grundstücksnutzer hätte jedenfalls dazu geführt, dass die personelle Umverteilung sich vorwiegend innerhalb der Gruppen der Grundstücksnutzer (nicht geförderter zu gefördertem sozialen Wohnbau) abgespielt hätte.

Die Analyse zeigt darüber hinaus, dass die Widmungsabgabe für sich allein genommen nur in Teilgebieten baulandmobilisierende Wirkungen aufgewiesen hätte. Vor allem in jenen Gebieten, wo aufgrund der (rationalen) Erwartung mittel- bis langfristig überdurchschnittlich steigender Bodenpreise das Problem der Bodenhortung am stärksten spürbar ist (strukturstarke bzw. nachfragestarke Regionen, Zentren), hätte die Widmungsabgabe nicht zu einer Baulandmobilisierung geführt. Dort hätten spekulativ denkende Grundstückseigentümer ihren Verkaufszeitpunkt nach wie vor an den prognostizierten Preisentwicklungen orientiert. Am ehesten hätte die Widmungsabgabe eine Mobilisierung in Gebieten mit stabilen oder nur moderat steigenden Bodenpreisen in Verbindung mit finanziellen Schieflagen der Grundeigentümer (also in strukturschwachen Randregionen) bewirkt. Dort allerdings hätte sie ohne entsprechende begleitende raumplanerische Maßnahmen sogar zu rascherer und verstärkter Zersiedelung geführt.

\section{Literatur}

Amt der Niederösterreichischen Landesregierung (2007): Motivenbericht zur 17. Novelle zum NÖ Raumordnungsgesetz 1976 (NÖ ROG 1976) vom 19. Juni 2007 (RU1-RO-2/018-2005). Sankt Pölten.

ARGE ALP - Arbeitsgemeinschaft Alpenländer (2000): Mobilisierung von Bauland in der ARGE ALP. Projektbericht. Innsbruck.

Bienert, S.; Funk, M. (Hrsg.) (2007): Immobilienbewertung Österreich. Wien.

Eekhoff, J. (1987): Wohnungs- und Bodenmarkt. Tübingen.

Evans, A. W. (2004): Economics and Land Use Planning. Oxford. 
Gewinn (2010): Grundstückspreise in Österreich - Baugrundpreise aus allen Gemeinden. Online unter: http://www.gewinn.com/immobilien/preisuebersichten (letzter Zugriff am 29.06.2011).

Glaeser, E. L.; Gyourko, J. (2003): The Impact of Building Restrictions on Housing Affordability. In: FRBNY Economic Policy Review 9, 2, 21-39.

Junker, T. (2010): Rechtliche Möglichkeiten und Grenzen einer Abschöpfung planungsbedingter Bodenwertsteigerungen durch Gemeinden. Dissertation an der Universität zu Köln. Online unter: http://kups.ub.uni-koeln.de/3069/1/Dissertation.pdf (letzter Zugriff am 29.06.2011).

Landesrechnungshof Tirol (2006): Tiroler Bodenfonds. Bericht des Landesrechnungshofes zum Tiroler Bodenfonds an den Landtag. Innsbruck.

Lechner, J. (2006): Der Planungswertausgleich - Theoretische und praktische Aspekte einer Bodenwertabschöpfung. In: Kurswechsel 3, 38-49.

Markstein, M. (2004): Instrumente und Strategien zur Baulandentwicklung und Baulandmobilisierung in Deutschland, Österreich und der Schweiz - ein methodischer Vergleich mit Entwicklungsvorschlägen für das Instrumentarium zur Baulandentwicklung in Deutschland. Dissertation an der Technischen Universität München. Online unter: http://tumb1.biblio.tu-muenchen.de/publ/ diss/bv/2004/markstein.pdf (letzter Zugriff am 29.06.2011).

Nindler, P. (2010): Baulandüberhang bleibt: 120 Hektar neu ausgewiesen. Online unter: http://www.tt.com/csp/cms/sites/tt/ Tirol/524556-2/bauland\%C3\%BCberhang-bleibt-120-hektarneu-ausgewiesen.csp (letzter Zugriff am 05.07.2011).

Oberhuber, A.; Amann, W.; Bauernfeind, S. (2005): Benchmarking Nachhaltigkeit in der Wohnbauförderung der Bundesländer. Ein Projektbericht im Rahmen der Programmlinie Impulsprogramm Nachhaltig Wirtschaften im Auftrag des Bundesministeriums für Verkehr, Innovation und Technologie. Wien.

Öggl, H. (2010): Flächenwidmungsplanung in Tirol 2008. Online unter: http://www.tirol.gv.at/fileadmin/www.tirol.gv.at/raumordnung/downloads/widmungsbilanz2008.pdf (letzter Zugriff am 29.06.2011)

Salvi, M. (2004): Ist eine höhere bauliche Dichte wünschenswert? Evidenz aus den Preisen von Einfamilienhäusern im Kanton Zürich. Online unter: http://www.cer.ethz.ch/resec/sgvs/001.pdf (letzter Zugriff am 29.06.2011).

Statistik Austria (2010a): Regionale Gesamtrechnungen. Online unter: http://www.statistik.at/web_de/statistiken/volkswirtschaftliche_gesamtrechnungen/regionale_gesamtrechnungen/index.html (letzter Zugriff am 05.07.2011).
Statistik Austria (2010b): Tourismus Bestandsstatistik: Entwicklung der Betriebe und Betten. Online unter: http://www.statistik. at/web_de/statistiken/tourismus/beherbergung/betriebe_betten/ index.html (letzter Zugriff am 29.06.2011).

Statistik Austria (2010c): Wohnbauraten nach Bundesländern. Online unter: http://www.statistik.at/web_de/statistiken/wohnen_und gebaeude/errichtung_von_gebaeuden_und_wohnungen/fertigstellungen/index.html (letzter Zugriff am 29.06.2011).

Statistik Austria (2010d): Wohnungsaufwand. Online unter: http:// www.statistik.at/web_de/statistiken/wohnen_und_gebaeude/ wohnungsaufwand/index.html (letzter Zugriff am 29.06.2011).

Statistik Austria (2010e): Jahresdurchschnittsbevölkerung seit 1961 nach Bundesland. Online unter: http://www.statistik.at/web_de/ statistiken/bevoelkerung/bevoelkerungsstand_und_veraenderung/bevoelkerung_im jahresdurchschnitt/index.html (letzter Zugriff am 29.06.2011).

Statistik Austria (2010f): Ergebnisse im Überblick Bevölkerungsprognose - Tirol. Online unter: http://www.statistik.at/web_de/ statistiken/bevoelkerung/demographische_prognosen/bevoelkerungsprognosen/index.html (letzter Zugriff am 29.06.2011).

Traurig, P. (2009): Are people cooperative? - A survey on experimental research of behaviour in social dilemma situations: motives, patterns and implications for policy-makers. Wien. Der Öffentliche Sektor - Forschungsmemoranden, 1-2/2009. Online unter: http://www.ifip.tuwien.ac.at/publ/oes/oes_pdf/oes_2009_1-2.pdf (letzter Zugriff am 29.06.2011).

TROG-Novelle (2010): Tiroler Raumordnungsgesetz - Novelle.Fassung vom 5. Juli 2010.

TROG-Novelle, Erläuternde Bemerkungen (2010): Tiroler Raumordnungsgesetz - Novelle. Entwurf einer Novelle zum Tiroler Raumordnungsgesetz 2006. Erläuternde Bemerkungen. Fassung vom 5. Juli 2010.

Waber, B. (2010): Mehrwertabgabe bei Einzonungen mobilisiert die Kantone. In: Neue Zürcher Zeitung vom 09.11.2010, 21.

Wieser, R. (2008): Marktbewertung struktureller Eigenschaften der Wiener Wohnbaulandgrundstücke, Mietwohnhäuser und Eigenheime. Wien. = IFIP Working Paper 2/2008. Online unter: http:// www.ifip.tuwien.ac.at/publikationen.htm (letzter Zugriff am 29.06.2011). 